\title{
Analytic and Approximate Solutions of the Space-Time Fractional Schrödinger Equations by Homotopy Perturbation Sumudu Transform Method
}

\author{
Sara H. M. Hamed, ${ }^{1,2}$ Eltayeb A. Yousif, ${ }^{2,3}$ and Arbab I. Arbab ${ }^{4}$ \\ ${ }^{1}$ Department of Mathematics, Faculty of Mathematical Sciences and Statistics, Alneelain University, 11121 Khartoum, Sudan \\ ${ }^{2}$ Department of Applied Mathematics, Faculty of Mathematical Sciences, University of Khartoum, 11111 Khartoum, Sudan \\ ${ }^{3}$ Department of Mathematics, Faculty of Science, Northern Border University, Arar 91431, Saudi Arabia \\ ${ }^{4}$ Department of Physics, Faculty of Science, University of Khartoum, 11111 Khartoum, Sudan
}

Correspondence should be addressed to Eltayeb A. Yousif; eltayebyousif@gmail.com

Received 2 May 2014; Revised 21 July 2014; Accepted 21 July 2014; Published 14 October 2014

Academic Editor: Douglas R. Anderson

Copyright (C) 2014 Sara H. M. Hamed et al. This is an open access article distributed under the Creative Commons Attribution License, which permits unrestricted use, distribution, and reproduction in any medium, provided the original work is properly cited.

A combination of homotopy perturbation method and Sumudu transform is applied to find exact and approximate solution of space and time fractional nonlinear Schrödinger equation. The fractional derivatives are described in the Caputo sense. The solutions are given in the form of convergent series with easily computable components. The results show that the method is effective and convenient for solving nonlinear differential equations of fractional order.

\section{Introduction}

Fractional calculus is a generalization of differentiations and integrations of integer order to arbitrary orders [17]. Fractional calculus has attracted much attention due to its appearance and numerous applications in science and engineering during the last decades. Many problems in physics, biology, and engineering are modulated in terms of fractional differential and integral equations such as acoustics, diffusion, signal processing, electrochemistry, systems identification, finance, fractional dynamics, nanotechnology, fluid dynamics, stochastic dynamical system, plasma physics and controlled thermonuclear fusion, nonlinear control theory, image processing, nonlinear biological systems, astrophysics, and maybe other physical phenomena [8-13]. Recently, there is a very comprehensive literature dealing with the problems of finding exact and approximate solutions of fractional differential equations. The solutions of fractional equations are investigated by many authors using powerful analytical methods. For example, the homotopy perturbation method [14-16], the Adomian decomposition method [17-19], the variational iteration method [20-22], the differential transform method [23-25], the fractional Riccati expansion method [26], the fractional subequation method $[27,28]$, the homotopy analysis method $[29,30]$, and the fractional complex transform [31]. Watugala [32] introduced Sumudu transform and used it in obtaining the solution of ordinary differential equations in control engineering problems. This method has been implemented by many authors in investigating various types of problems [3340]. The homotopy perturbation Sumudu transform method (HPSTM) is a combination of the Sumudu transform method and homotopy perturbation method. It is applied to solve numerous linear and nonlinear partial differential equations [41-47].

Schrödinger equation is one of the most important models in mathematical physics; it arises in many physical systems with applications to numerous fields [48] such as nonlinear optics [49], dynamics of accelerators [50], mean-field theory of Bose-Einstein condensates [51, 52], and plasma physics 
[53]. The fractional Schrödinger equation is a developing part of quantum physics which studies nonlocal quantum phenomena. Naber [54] studied time fractional Schrödinger equation in sense of Caputo derivative. Wang and $\mathrm{Xu}$ [55] generalized the linear Schrödinger equation to space-time fractional one and studied the problem by using integral transform technique. Jiang [56] obtained the time dependent solutions in terms of $\mathrm{H}$-function to a linear space-time fractional Schrödinger equation containing a nonlocal term. Ford et al. [57] introduced a numerical method to solve a linear fractional Schrödinger equation in the case where the space has dimension two; they obtained the stability conditions for a finite difference scheme. In recent years, nonlinear fractional Schrödinger equation has attracted several researchers. The existence and uniqueness of the global solution to the periodic boundary value problem of fractional nonlinear Schrödinger equations are proved based on energy method [58] and Faedo-Galërkin method [59]. Analytical and numerical methods have been investigated for time fractional nonlinear Schrödinger equation [18, 60-62]. Very few theoretical and numerical analyses have been carried out for nonlinear Schrödinger equations with both space and time fractional derivatives. Herzallah and Gepreel [19] constructed an approximate solution for the cubic nonlinear fractional Schrödinger equation with time and space fractional derivatives using Adomian decomposition method. Hemida et al. [29] used a homotopy analysis method to construct approximate solutions for the space-time fractional nonlinear Schrödinger equation.

In this paper, we applied the homotopy perturbation Sumudu transform method (HPSTM) to obtain the analytical exact and approximate solutions for the fractional Schrödinger equation with space and time fractional derivatives of the form

$$
\begin{aligned}
i D_{t}^{\alpha} U(x, t)= & c_{1} D_{x}^{\beta} U(x, t)+V(x) U(x, t) \\
& +c_{2} U(x, t)|U(x, t)|^{2}, \quad U(x, 0)=f(x),
\end{aligned}
$$

where $\alpha$ and $\beta$ are parameters describing the order of the time and space fractional derivatives of $U(x, t)$, respectively, and they satisfy $0<\alpha \leq 1,1<\beta \leq 2, t>0, V(x)$ is the trapping potential, $c_{1}, c_{2}$ are constants, and $f(x)$ is the initial condition [19]. The fractional derivatives are considered in the Caputo sense. In the case of $\alpha=1$ and $\beta=2$, (1) reduces to the classical Schrödinger equation. The solution of (1) is obtained for linear case when $c_{2}=0$ and nonlinear case when $c_{2} \neq 0$. Moreover, this method is applied in approximating the solution of the problem in the case of nonzero trapping potential when $V(x) \neq 0$.

The rest of this work is organized as follows. In Section 2, we provide some preliminaries. Section 3 introduces the concept of homotopy perturbation method, while Section 4 gives the Sumudu transform. The homotopy perturbation Sumudu transform method (HPSTM) is analyzed in Section 5. Applications of numerical examples are provided in Section 6. The conclusions are given in Section 7.

\section{Preliminaries}

Definition 1. The Caputo fractional derivative of order $\alpha>0$ of a function $f(x), x>0$, is defined by $[4,10]$

$$
\begin{aligned}
& D_{x}^{\alpha} f(x) \\
& = \begin{cases}\frac{1}{\Gamma(n-\alpha)} \int_{0}^{x}(x-t)^{n-\alpha-1} f^{(n)}(t) d t, & n-1<\alpha \leq n \in \mathbb{N} \\
\frac{d^{n}}{d x^{n}} f(x), & \alpha=n \in \mathbb{N},\end{cases}
\end{aligned}
$$

where $D_{x}^{\alpha}$ is called the Caputo derivative operator.

Definition 2. The Mittag-Leffler function with two parameters is defined by $[63,64]$

$$
E_{\alpha, \beta}(x)=\sum_{n=0}^{\infty} \frac{x^{n}}{\Gamma(\alpha n+\beta)}, \quad \alpha, \beta \geq 0 .
$$

The Mittag-Leffler function $E(x, m, a)$ is expressed in terms of formula (3) as

$$
E(x, m, a)=x^{m} E_{1, m+1}(a x) .
$$

Note 1. The following results are used further in this paper.

From Definition 2 and formula (4), these functions are directly obtained:

$$
E(x,-m, \lambda)=x^{-m} E_{1,1-m}(\lambda x)=\lambda^{m} e^{\lambda x}, \quad m=0,1,2, \ldots
$$

The special type of Mittag-Leffler function $E_{1 / 2,1}(a \sqrt{t})$ is given by [58]

$$
E_{1 / 2,1}(a \sqrt{x})=e^{a^{2} t} \operatorname{erfc}(-a \sqrt{x}),
$$

where $\operatorname{erfc}$ is a complementary error function.

The fractional derivatives of Mittag-Leffler function is given by $[10,64]$

$$
D_{x}^{\gamma}\left[x^{\beta-1} E_{\alpha, \beta}\left(a x^{\alpha}\right)\right]=x^{\beta-\gamma-1} E_{\alpha, \beta-\gamma}\left(a x^{\alpha}\right), \quad \gamma \geq 0 .
$$

\section{Homotopy Perturbation Method}

To illustrate the concept of homotopy perturbation method, we consider the nonlinear differential equation,

$$
L(U)+N(U)=f(r), \quad r \in \Omega,
$$

with the boundary conditions,

$$
B\left(U, \frac{\partial U}{\partial n}\right)=0, \quad r \in \Gamma,
$$

where $L$ is a linear operator, $N$ is nonlinear operator, $B$ is boundary operator, $\Gamma$ is the boundary of the domain $\Omega$, and $f(r)$ is a known analytic function. 
He's homotopy perturbation technique [65-67] defines the homotopy $v(r, p): \Omega \times[0,1] \rightarrow R$ which satisfies

$$
\begin{aligned}
H(v, p) & =(1-p)\left[L(v)-L\left(U_{0}\right)\right]+p[L(v)+N(v)-f(r)] \\
& =0
\end{aligned}
$$

or

$$
\begin{aligned}
H(v, p) & =L(v)-L\left(U_{0}\right)+p L\left(U_{0}\right)+p[N(v)-f(r)] \\
& =0
\end{aligned}
$$

where $r \in \Omega, p \in[0,1]$ is an impending parameter, and $U_{0}$ is an initial approximation which satisfies the boundary condition. The basic assumption is that the solution of (10) and (11) can be expressed as power series in $p$ as follows:

$$
v=v_{0}+p v_{1}+p^{2} v_{2}+\cdots
$$

The approximate solution of (8) is given by

$$
U=\lim _{p \rightarrow 1} v=v_{0}+v_{1}+v_{2}+\cdots
$$

\section{Sumudu Transform}

Consider functions in the set $A$ that are defined by

$$
\begin{gathered}
A=\left\{f(t): \exists M, \tau_{1}, \tau_{2}>0, \text { such }|f(t)|<M e^{|t| / \tau_{j}}\right. \\
\text { if } \left.t \in(-1)^{j} \times[0, \infty)\right\},
\end{gathered}
$$

where $M$ is a constant and must be finite and $\tau_{1}$ and $\tau_{2}$ need not simultaneously exist and each may be finite. The Sumudu transform is defined by [32]

$$
G(u)=S(f(t))=\int_{0}^{\infty} f(u t) e^{-t} d t, \quad u \in\left(-\tau_{1}, \tau_{2}\right)
$$

The Sumudu transform was shown to be the theoretical dual of the Laplace transform. For the details of the relationship between Sumudu and the Laplace transforms and the comparison between the two transformations, see [33-37].

Definition 3. The Sumudu transform of fractional order derivative is defined by $[45,46]$

$$
\begin{array}{r}
S\left[D_{x}^{\alpha} f(x)\right]=\frac{1}{u^{\alpha}} S[f(x)]-\sum_{k=0}^{n-1} \frac{1}{u^{\alpha-k}}\left[f^{(k)}(x)\right]_{x=0}, \\
n-1<\alpha \leq n, \quad n \in \mathbb{N} .
\end{array}
$$

\section{Homotopy Perturbation Sumudu Transform Method}

In this section, we implement the homotopy perturbation Sumudu transform method to space-time cubic nonlinear fractional Schrödinger equation given by (1). By applying
Sumudu transform on both sides of (1) with respect to $t$, we get the following:

$$
\begin{aligned}
& S\left(i D_{t}^{\alpha} U(x, t)\right) \\
& =S\left(c_{1} D_{x}^{\beta} U(x, t)+V(x) U(x, t)+c_{2} U(x, t)|U(x, t)|^{2}\right), \\
& \frac{i}{u^{\alpha}} S(U(x, t))-i \sum_{k=0}^{n-1} \frac{1}{u^{\alpha-k}} \frac{\partial^{k} U(x, 0)}{\partial t^{k}} \\
& =S\left(c_{1} D_{x}^{2 \beta} U(x, t)+V(x) U(x, t)+c_{2} U(x, t)|U(x, t)|^{2}\right), \\
& S(U(x, t)) \\
& =\sum_{k=0}^{n-1} u^{k} f_{k}(x)-i u^{\alpha}\left(S \left(c_{1} D_{x}^{\beta} U(x, t)+V(x) U(x, t)\right.\right. \\
& \left.\left.\quad+c_{2} U(x, t)|U(x, t)|^{2}\right)\right) .
\end{aligned}
$$

Taking the inverse Sumudu transform to (17), we have

$$
\begin{aligned}
U(x, t)= & \sum_{k=0}^{n-1} \frac{t^{k} f_{k}(x)}{\Gamma(k+1)} \\
& -i S^{-1}\left(u ^ { \alpha } \left(S \left(c_{1} D_{x}^{\beta} U(x, t)+V(x) U(x, t)\right.\right.\right. \\
& \left.\left.\left.+c_{2} U(x, t)|U(x, t)|^{2}\right)\right)\right) .
\end{aligned}
$$

Application of the homotopy perturbation method to (18) yields

$$
\begin{gathered}
(1-P)(U(x, t)-U(x, 0)) \\
+P\left(U(x, t)-\sum_{k=0}^{n-1} \frac{t^{k} f_{k}(x)}{\Gamma(k+1)}\right. \\
+i S^{-1}\left(u ^ { \alpha } \left(S \left(c_{1} D_{x}^{\beta} U(x, t)+V(x) U(x, t)\right.\right.\right. \\
\left.\left.\left.\left.+c_{2} U(x, t)|U(x, t)|^{2}\right)\right)\right)\right)=0 \\
U(x, t)=U(x, 0)+P \sum_{k=1}^{n-1} \frac{t^{k} f_{k}(x)}{\Gamma(k+1)} \\
-i P S^{-1}\left(u ^ { \alpha } \left(S \left(c_{1} D_{x}^{\beta} U(x, t)+V(x) U(x, t)\right.\right.\right. \\
\left.\left.\left.\quad+c_{2} U(x, t)|U(x, t)|^{2}\right)\right)\right) .
\end{gathered}
$$

Let

$$
U(x, t)=\sum_{n=0}^{\infty} P^{n} U_{n}(x, t), \quad U(x, 0)=U_{0}(x, t) .
$$


Substituting (20) into (19), we get

$$
\begin{aligned}
& \sum_{n=0}^{\infty} P^{n} U_{n}(x, t) \\
& =U_{0}(x, t)+P \sum_{k=1}^{n-1} \frac{t^{k} f_{k}(x)}{\Gamma(k+1)} \\
& -i P S^{-1}\left(u ^ { \alpha } \left(S\left(c_{1} D_{x}^{\beta} \sum_{n=0}^{\infty} P^{n} U_{n}(x, t)\right)\right.\right. \\
& +V(x) S\left(\sum_{n=0}^{\infty} P^{n} U_{n}(x, t)\right) \\
& \left.\left.+S\left(\sum_{n=0}^{\infty} P^{n} H_{n}(U(x, t))\right)\right)\right)
\end{aligned}
$$

where

$$
\begin{array}{r}
H_{n}\left(U_{0}, U_{1}, \ldots, U_{n}\right)=\frac{1}{n !} \frac{\partial^{n}}{\partial p^{n}}\left[N\left(\sum_{i=0}^{\infty} p^{i} U_{i}\right)\right]_{p=0}, \\
n=0,1,2, \ldots,
\end{array}
$$

and then

$$
\begin{aligned}
P^{0}: U_{0}(x, t)=f_{0}(x)=f(x), & \\
P^{1}: U_{1}(x, t)=\sum_{k=1}^{n-1} \frac{t^{k} f_{k}(x)}{\Gamma(k+1)} & \\
& -i S^{-1}\left(u ^ { \alpha } \left(S\left(c_{1} D_{x}^{\beta} U_{0}(x, t)\right)\right.\right. \\
& +V(x) S\left(U_{0}(x, t)\right) \\
& \left.\left.+S\left(H_{0}(U(x, t))\right)\right)\right) \\
P^{2}: U_{2}(x, t)=-i S^{-1}\left(u^{\alpha}(\right. & S\left(c_{1} D_{x}^{\beta} U_{1}(x, t)\right) \\
& +V(x) S\left(U_{1}(x, t)\right) \\
& \left.\left.+S\left(H_{1}(U(x, t))\right)\right)\right), \\
P^{3}: U_{3}(x, t)=-i S^{-1}\left(u^{\alpha}(\right. & S\left(c_{1} D_{x}^{\beta} U_{2}(x, t)\right) \\
& +V(x) S\left(U_{2}(x, t)\right) \\
& \left.\left.+S\left(H_{2}(U(x, t))\right)\right)\right),
\end{aligned}
$$

The HPMST assumes a series solution to (1) in the form

$$
U=U_{0}+U_{1}+U_{2}+\cdots=\sum_{n=1}^{\infty} U_{n} .
$$

$$
\begin{gathered}
=\frac{i t^{\alpha}}{\Gamma(\alpha+1)} e^{i x}, \\
U_{2}(x, t)=i S^{-1}\left(u^{\alpha} S\left(\frac{\partial^{2} U_{1}}{\partial x^{2}}+2\left(2 U_{0} U_{1} \overline{U_{0}}+U_{0}{ }^{2} \overline{U_{1}}\right)\right)\right) \\
=i S^{-1}\left(u ^ { \alpha } S \left(-\frac{i t^{\alpha}}{\Gamma(\alpha+1)} e^{i x}\right.\right. \\
+2\left(2 e^{i x}\left(\frac{i t^{\alpha}}{\Gamma(\alpha+1)} e^{i x}\right) e^{-i x}\right. \\
\left.\left.\left.+e^{2 i x}\left(-\frac{i t^{\alpha}}{\Gamma(\alpha+1)} e^{-i x}\right)\right)\right)\right) \\
=i S^{-1}\left(u^{\alpha} S\left(\frac{i t^{\alpha}}{\Gamma(\alpha+1)} e^{i x}\right)\right)=-\frac{t^{2 \alpha}}{\Gamma(2 \alpha+1)} e^{i x} .
\end{gathered}
$$




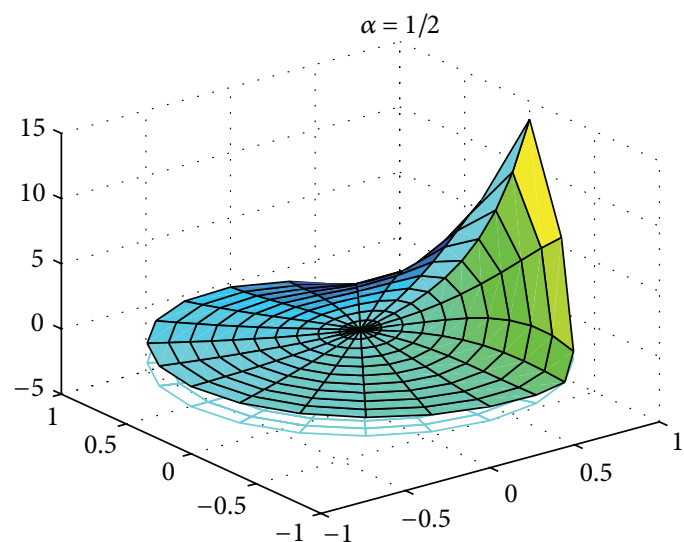

(a)

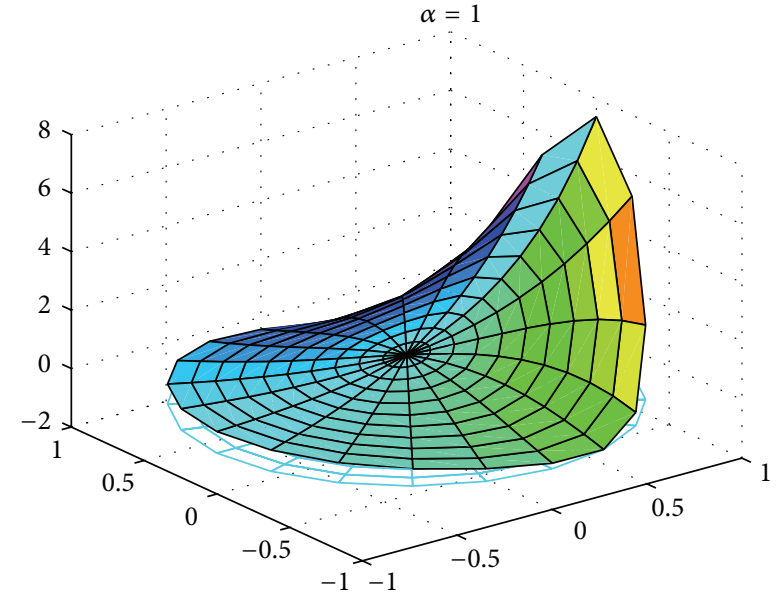

(b)

Figure 1: The surface plot of the solution $U(x, t)$ of Example 4: (a) $\alpha=0.5$, (b) $\alpha=1$.

Substituting $U_{0}(x, t), U_{1}(x, t), U_{2}(x, t), \ldots$ into (24) yields the solution

$$
\begin{aligned}
U(x, t) & =e^{i x}+\frac{i t^{\alpha}}{\Gamma(\alpha+1)} e^{i x}-\frac{t^{2 \alpha}}{\Gamma(2 \alpha+1)} e^{i x}+\cdots \\
& =e^{i x} E_{\alpha, 1}\left(i t^{\alpha}\right)
\end{aligned}
$$

Remark 5. If $\alpha \rightarrow 1 / 2$, then the solution is given by $U(x, t)=$ $e^{i x} E_{1 / 2,1}(i \sqrt{t})=e^{i x-t} \operatorname{erf} c(-i \sqrt{t})$. The solution for the classical nonlinear Schrödinger equation is obtained as special case as $\alpha \rightarrow 1$, by $U(x, t)=e^{i x} E_{1,1}(i t)=e^{i(x+t)}$

Figure 1 shows the solution $U(x, t)$ of Example 4, Figure 1(a) the solution obtained for $\alpha=0.5$, and Figure 1(b) that obtained for the value of $\alpha=1$ (the solution of classical nonlinear Schrödinger equation).

Example 6. Consider the following linear space-time fractional Schrödinger equation:

$$
i D_{t}^{\alpha} U(x, t)=c D_{x}^{\beta} U(x, t),
$$

$0<\alpha \leq 1,1<\beta \leq 2$, and $c$ is a constsnt,

subject to the initial condition

$$
U(x, 0)=e^{i x} .
$$

By using (21) and (23), we have

$$
\begin{aligned}
& i \sum_{n=0}^{\infty} P^{n} U_{n}(x, t) \\
& \quad=i U_{0}(x, t)+c P S^{-1}\left(u^{\alpha}\left(S\left(\frac{\partial^{\beta}}{\partial x^{\beta}} \sum_{n=0}^{\infty} P^{n} U_{n}(x, t)\right)\right)\right)
\end{aligned}
$$

and then

$$
\begin{aligned}
& P^{0}: U_{0}(x, t)=e^{i x} \\
& P^{1}: U_{1}(x, t)=-i c S^{-1}\left(u^{\alpha} S\left(\frac{\partial^{\beta} U_{0}}{\partial x^{\beta}}\right)\right) \\
&=-i c S^{-1}\left(u^{\alpha} S\left(\frac{\partial^{\beta}}{\partial x^{\beta}} e^{i x}\right)\right) \\
&=\frac{i c t^{\alpha}}{\Gamma(\alpha+1)} x^{2-\beta} E_{1,3-\beta}(i x) \\
&=\frac{i c t^{\alpha}}{\Gamma(\alpha+1)} E(x, 2-\beta, i)
\end{aligned}
$$

$P^{2}: U_{2}(x, t)$

$$
\begin{aligned}
& =-i c S^{-1}\left(u^{\alpha} S\left(\frac{\partial^{\beta} U_{1}}{\partial x^{\beta}}\right)\right) \\
& =-i c S^{-1}\left(u^{\alpha} S\left(\frac{i c t^{\alpha}}{\Gamma(\alpha+1)} D_{x}^{\beta}(E(x, 2-\beta, i))\right)\right) \\
& =\frac{c^{2} t^{2 \alpha}}{\Gamma(2 \alpha+1)} x^{2-2 \beta} E_{1,3-2 \beta}(i x) \\
& =\frac{c^{2} t^{2 \alpha}}{\Gamma(2 \alpha+1)} E(x, 2-2 \beta, i),
\end{aligned}
$$

$$
\begin{aligned}
P^{3} & : U_{3}(x, t) \\
& =-i c S^{-1}\left(u^{\alpha} S\left(\frac{\partial^{\beta} U_{2}}{\partial x^{\beta}}\right)\right) \\
& =-i c S^{-1}\left(u^{\alpha} S\left(\frac{c^{2} t^{2 \alpha}}{\Gamma(2 \alpha+1)}\left(\frac{\partial^{\beta}}{\partial x^{\beta}} E(x, 2-2 \beta, i)\right)\right)\right)
\end{aligned}
$$




$$
=-\frac{i c^{3} t^{3 \alpha}}{\Gamma(3 \alpha+1)} E(x, 2-3 \beta, i) .
$$

Substituting $U_{0}(x, t), U_{1}(x, t), U_{2}(x, t), \ldots$ into (24) gives the series solution as

$$
\begin{aligned}
U(x, t)= & e^{i x}+\frac{i c t^{\alpha}}{\Gamma(\alpha+1)} E(x, 2-\beta, i) \\
& +\frac{c^{2} t^{2 \alpha}}{\Gamma(2 \alpha+1)} E(x, 2-2 \beta, i) \\
& -\frac{i c^{3} t^{3 \alpha}}{\Gamma(3 \alpha+1)} E(x, 2-3 \beta, i)+\cdots \\
= & e^{i x}+\frac{i c t^{\alpha}}{\Gamma(\alpha+1)} E(x, 2-\beta, i) \\
& -\frac{\left(i c t^{\alpha}\right)^{2}}{\Gamma(2 \alpha+1)} E(x, 2-2 \beta, i) \\
& +\frac{\left(i c t^{\alpha}\right)^{3}}{\Gamma(3 \alpha+1)} E(x, 2-3 \beta, i)+\cdots \\
= & e^{i x}+\sum_{k=1}^{\infty}(-1)^{k+1} \frac{\left(i c t^{\alpha}\right)^{k}}{\Gamma(k \alpha+1)} E(x, 2-\beta k, i) .
\end{aligned}
$$

Remark 7. In (34), if we let $\alpha \rightarrow 1, \beta \rightarrow 2$, and $c=1$, using the result given by formula (5), then we get the solution of the classical equation as

$$
\begin{aligned}
U_{\text {clas }}(x, t) & =e^{i x}+\sum_{k=1}^{\infty}(-1)^{k+1} \frac{(i t)^{k}}{\Gamma(k+1)} E(x, 2-2 k, i) \\
& =e^{i x}+\sum_{k=1}^{\infty} \frac{(i t)^{k}}{\Gamma(k+1)} e^{i x}=e^{i(x+t)} .
\end{aligned}
$$

Remark 8. Let $\widetilde{U}(x, t)$ be the solution when $\alpha \rightarrow 1 / 2, \beta \rightarrow$ $3 / 2$, and $c=1$; then, from (34), we have

$$
\begin{aligned}
\widetilde{U}(x, t)= & e^{i x}+\sum_{k=1}^{\infty}(-1)^{k+1} \frac{(i \sqrt{t})^{k}}{\Gamma((k / 2)+1)} E\left(x, 2-\frac{3}{2} k, i\right) \\
= & {\left[e^{i x}+\sum_{k=1}^{\infty}(-1)^{k+1} \frac{t^{k}}{\Gamma(k+1)} E(x, 2-3 k, i)\right] } \\
& +\left[\sum_{k=1}^{\infty} \frac{(i \sqrt{t})^{k}}{\Gamma(k+(1 / 2))} E\left(x, \frac{1-6 k}{2}, i\right)\right]
\end{aligned}
$$

$$
\begin{aligned}
= & {\left[e^{i x}+\sum_{k=1}^{\infty}\left(\frac{(i t)^{k}}{\Gamma(k+1)}\right) e^{i x}\right] } \\
& +\left[\sum_{k=1}^{\infty} \frac{(i \sqrt{t})^{k}}{\Gamma(k+(1 / 2))} E\left(x, \frac{1-6 k}{2}, i\right)\right] \\
= & U_{\text {clas }}(x, t)+\left[\sum_{k=1}^{\infty} \frac{(i \sqrt{t})^{k}}{\Gamma(k+(1 / 2))} E\left(x, \frac{1-6 k}{2}, i\right)\right] .
\end{aligned}
$$

It is noted that the function $\sum_{k=1}^{\infty}\left((i \sqrt{t})^{k} / \Gamma(k+\right.$ $(1 / 2))) E(x,((1-6 k) / 2), i)$ represents the variation between the two solutions $U_{\text {clas }}(x, t)$ and $\widetilde{U}(x, t)$ given by (35) and (36), respectively.

Figure 2 compares the real part of the solution of Example 6. In Figure 2(a) we have the solution obtained for the value of $\alpha=0.5, \beta=1.5$ and in Figure 2(b) for the value of $\alpha=1, \beta=2$ (the solution of classical nonlinear Schrödinger equation). Figure 3 compares the imaginary part of the solution of Example 6. In Figure 3(a) we have the solution obtained for the value of $\alpha=0.5, \beta=1.5$ and in Figure 3(b) the solution obtained for the value of $\alpha=1, \beta=2$ (the solution of classical nonlinear Schrödinger equation).

Example 9. Consider the space-time fractional nonlinear Schrödinger equation:

$$
i D_{t}^{\alpha} U+D_{x}^{\beta} U+2\left|U^{2}\right| U=0, \quad 0<\alpha \leq 1,1<\beta \leq 2,
$$

subject to the initial condition

$$
U(x, 0)=e^{i x} .
$$

By applying (21)-(23), we have

$$
\begin{aligned}
& i \sum_{n=0}^{\infty} P^{n} U_{n}(x, t) \\
& =i U_{0}(x, t)-P S^{-1} \\
& \times\left(u^{\alpha}\left(S\left(\frac{\partial^{\beta}}{\partial x^{\beta}} \sum_{n=0}^{\infty} P^{n} U_{n}(x, t)+2 \sum_{n=0}^{\infty} P^{n} H_{n}(U(x, t))\right)\right)\right), \\
& P^{0}: U_{0}(x, t)=e^{i x}, \\
& P^{1}: U_{1}(x, t)=i S^{-1}\left(u^{\alpha} S\left(\frac{\partial^{\beta} U_{0}}{\partial x^{\beta}}+2 U_{0}{ }^{2} \overline{U_{0}}\right)\right) \\
& \quad=\frac{-i t^{\alpha}}{\Gamma(\alpha+1)}\left(E(x, 2-\beta, i)-2 e^{i x}\right) \\
& P^{2}: U_{2}(x, t) \\
& \left.=i S^{-1}\left(\frac{\partial^{\beta} e^{i x}}{\partial x^{\beta}}+2 e^{2 i x} e^{-i x}\right)\right) \\
& \left.\left(\frac{\partial^{\beta} U_{1}}{\partial x^{\beta}}+2\left(2 U_{0} U_{1} \overline{U_{0}}+U_{0}{ }^{2} \overline{U_{1}}\right)\right)\right)
\end{aligned}
$$




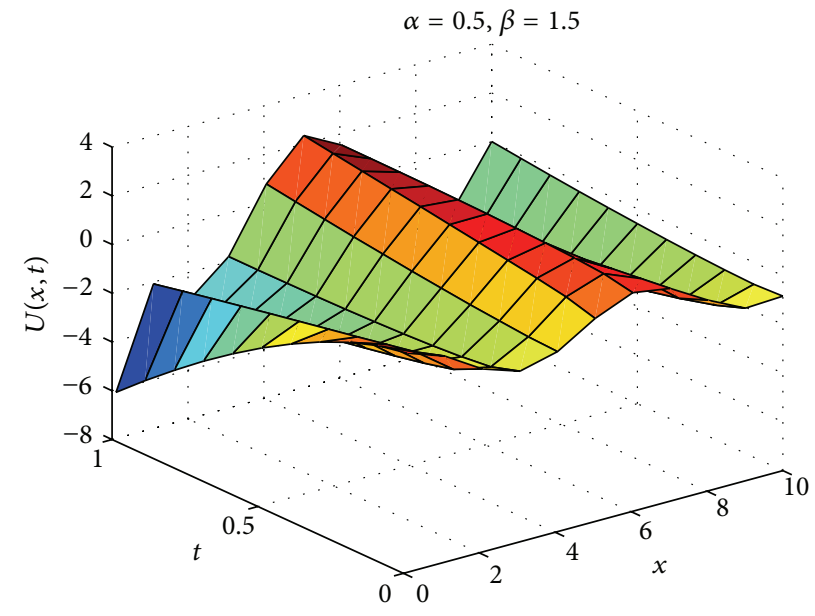

(a)

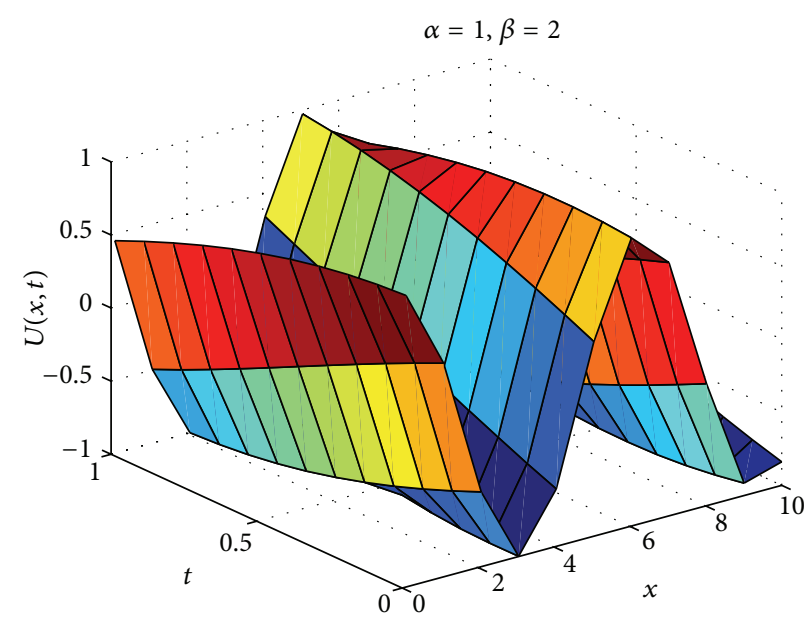

(b)

Figure 2: The surface plot of the real part of the solution $U(x, t)$ of Example 6 : (a) $\alpha=0.5, \beta=1.5$, (b) $\alpha=1, \beta=2$.

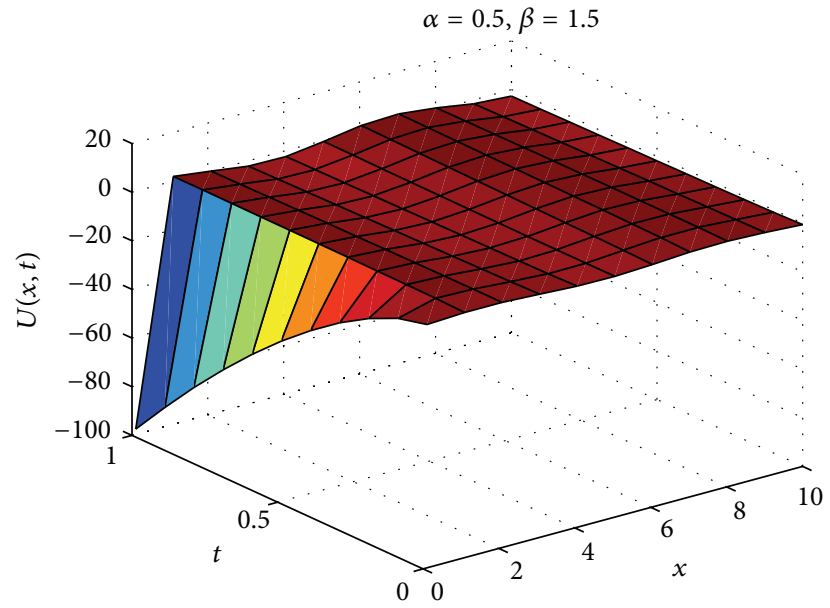

(a)

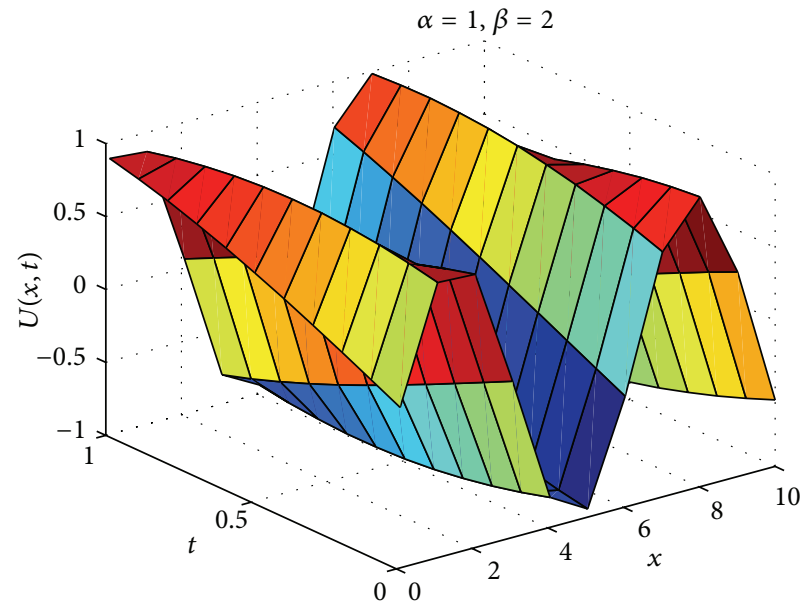

(b)

FIgURE 3: The surface plot of the imaginary part of the solution $U(x, t)$ of Example 6 : (a) $\alpha=0.5, \beta=1.5$, (b) $\alpha=1, \beta=2$.

$$
\begin{aligned}
=i S^{-1}\left(u ^ { \alpha } S \left(D_{x}^{\beta}\left(\frac{i t^{\alpha}}{\Gamma(\alpha+1)}\left(-E(x, 2-\beta, i)+2 e^{i x}\right)\right)\right.\right. \\
+2\left(2 e^{i x}\left(\frac{i t^{\alpha}}{\Gamma(\alpha+1)}\left(-E(x, 2-\beta, i)+2 e^{i x}\right)\right)\right. \\
\left.\left.\left.\quad \times e^{-i x}+e^{2 i x} \overline{U_{1}}\right)\right)\right) \\
=\frac{t^{2 \alpha}}{\Gamma(2 \alpha+1)}(E(x, 2-2 \beta, i)+6 E(x, 2-\beta, i) \\
\left.-2 e^{2 i x} E(x, 2-\beta,-i)-4 e^{i x}\right) .
\end{aligned}
$$

Substituting $U_{0}(x, t), U_{1}(x, t), U_{2}(x, t), \ldots$ into $(24)$, we obtain the following approximate solution to (37) and (38):

$$
\begin{aligned}
U_{\text {app }}(x, t)=e^{i x}-\frac{i t^{\alpha}}{\Gamma(\alpha+1)}\left(E(x, 2-\beta, i)-2 e^{i x}\right) \\
+\frac{t^{2 \alpha}}{\Gamma(2 \alpha+1)}(E(x, 2-2 \beta, i)+6 E(x, 2-\beta, i) \\
\left.-2 e^{2 i x} E(x, 2-\beta,-i)-4 e^{i x}\right)
\end{aligned}
$$

Remark 10. If $\alpha \rightarrow 1$ and $\beta \rightarrow 2$, then the solution given by (40) converged to $U_{\text {clas }}(x, t)=e^{i(x+t)}$, which is the solution of the classical equation.

Figure 4 shows the surface plot of the approximate solution of Example 9 for the values of $\alpha=0.5, \beta=1.5$. In 


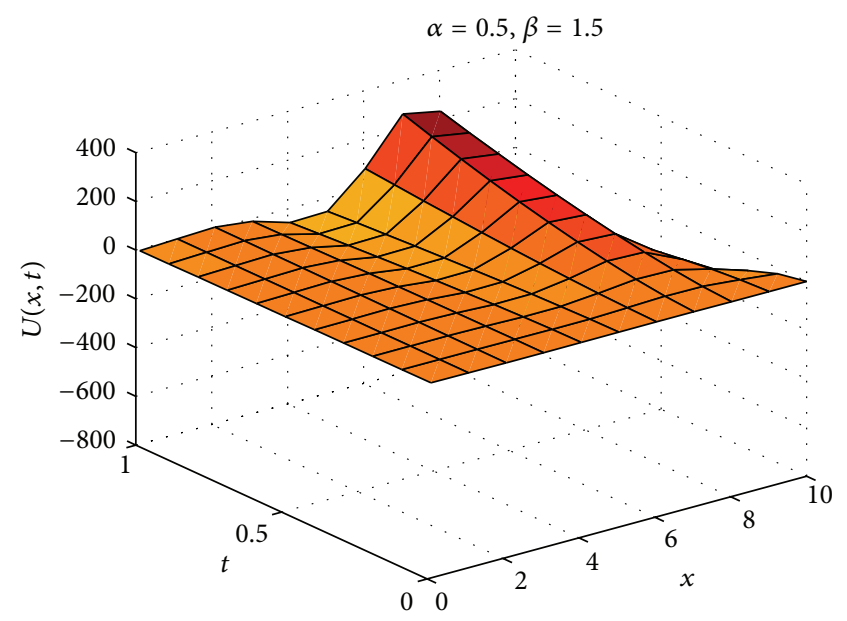

(a)

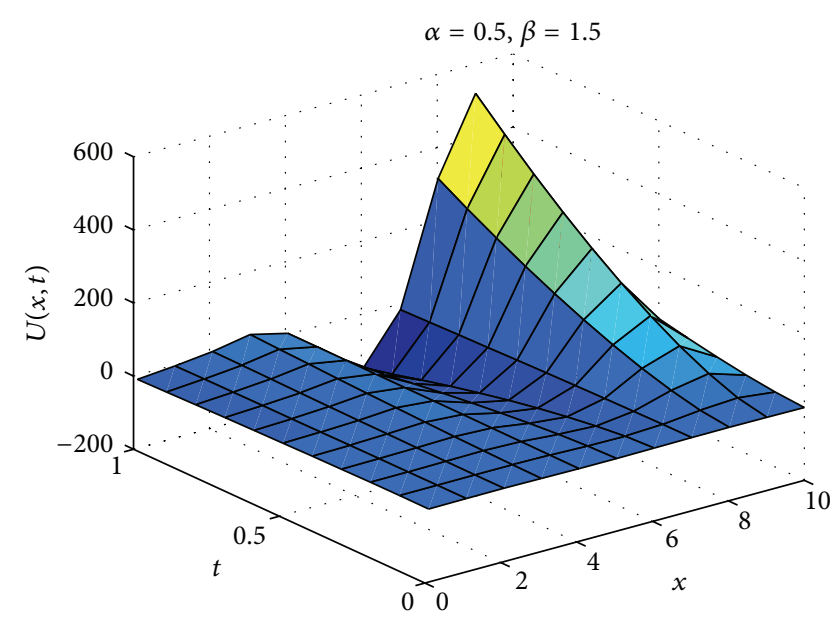

(b)

FIGURE 4: The surface plot of the solution $U_{\text {app }}(x, t)$ of Example 9 for the values of $\alpha=0.5, \beta=1.5$. (a) The real part of the solution and (b) the imaginary parts of the solution.

Figure 4(a) the graph is given for the real part of the solution and in Figure 4(b) it is given for the imaginary part of the solution.

Table 1 shows the comparison of the absolute approximate solution of Example 9 between the homotopy analysis method [29], the homotopy perturbation Sumudu transform used in this paper, and the exact solution. It shows that an approximate solution obtained by HPSTM is in perfect agreement with the exact solution for $\alpha=1, \beta=2$; it is noted that the absolute error between HPSTM and the exact solution is sufficiently small compared with the homotopy analysis method. Furthermore, the approximate solutions for the values of $\alpha=0.9, \beta=1.8$ obtained by the HPSTM and homotopy analysis method are compared. Clearly, the results obtained by HPSTM are closer to the exact solution than the homotopy analysis method.

Example 11. Consider the space-time fractional nonlinear Schrödinger equation with nonzero trapping potential:

$$
\begin{array}{r}
i D_{t}^{\alpha} U+\frac{1}{2} D_{x}^{\beta} U-U \cos ^{2} x-\left|U^{2}\right| U=0, \\
0<\alpha \leq 1, \quad 1<\beta \leq 2,
\end{array}
$$

subject to the initial condition

$$
U(x, 0)=\sin x
$$

By using (21)-(23), we get

$$
\begin{aligned}
i \sum_{n=0}^{\infty} P^{n} U_{n}(x, t) & \\
= & i U_{0}(x, t)-P S^{-1} \\
& \times\left(u ^ { \alpha } \left(S \left(\frac{1}{2} \frac{\partial^{\beta}}{\partial x^{\beta}} \sum_{n=0}^{\infty} P^{n} U_{n}(x, t)\right.\right.\right.
\end{aligned}
$$

$$
\begin{gathered}
-\cos ^{2} x \sum_{n=0}^{\infty} P^{n} U_{n}(x, t) \\
\left.\left.\left.-\sum_{n=0}^{\infty} P^{n} H_{n}(U(x, t))\right)\right)\right), \\
P^{0}: U_{0}(x, t)=\sin x
\end{gathered}
$$

$P^{1}: U_{1}(x, t)$

$$
\begin{aligned}
= & i S^{-1}\left(u^{\alpha} S\left(\frac{1}{2} \frac{\partial^{\beta} U_{0}}{\partial x^{\beta}}-U_{0} \cos ^{2} x-H_{0}(U(x, t))\right)\right) \\
= & i S^{-1}\left(u^{\alpha} S\left(\frac{1}{2} \frac{\partial^{\beta}}{\partial x^{\beta}} \sin x-\sin x \cos ^{2} x-\sin ^{3} x\right)\right) \\
= & \frac{t^{\alpha}}{\Gamma(\alpha+1)} \\
& \times\left(\left(\frac{i}{2}\right)^{2}(E(x, 2-\beta, i)-E(x, 2-\beta,-i))-i \sin x\right) \\
P^{2}: & U_{2}(x, t) \\
= & i S^{-1}\left(u^{\alpha} S\left(\frac{1}{2} \frac{\partial^{\beta} U_{1}}{\partial x^{\beta}}-U_{1} \cos ^{2} x-H_{1}(U(x, t))\right)\right)
\end{aligned}
$$

where

$$
\begin{aligned}
& \frac{1}{2} \frac{\partial^{\beta} U_{1}}{\partial x^{\beta}}-U_{1} \cos ^{2} x \\
& =\frac{t^{\alpha}}{\Gamma(\alpha+1)} \\
& \quad \times\left(\left(\left(\frac{i}{2}\right)^{2}(E(x, 2-\beta, i)-E(x, 2-\beta,-i))-i \sin x\right)\right.
\end{aligned}
$$


TABLE 1: Compare the absolute solution between the homotopy analysis method, the homotopy perturbation Sumudu transform, and the exact solution of Example 9 for $0<x<1,0<t<1$.

\begin{tabular}{lcccccc}
\hline \multicolumn{2}{c}{$\alpha=0.9, \beta=1.8$} & & \multicolumn{3}{c}{$\alpha=1, \beta=2$} \\
$U_{\text {HAM }}$ & $U_{\text {HPSTM }}$ & $U_{\text {HAM }}$ & $U_{\text {HPSTM }}$ & $U_{\text {clas }}$ & abs $\left|U_{\text {clas }}-U_{\text {HAM }}\right|$ & 0.0000 \\
1.0000 & 1.0000 & 1.0000 & 1.0000 & 1.0000 & 0.0002 & 0.0000 \\
1.0087 & 0.9682 & 1.0002 & 1.0000 & 1.0000 & 0.0032 & 0.0002 \\
1.0159 & 0.9528 & 1.0032 & 1.0002 & 1.0000 & 0.0161 & 0.0010 \\
1.0323 & 0.9539 & 1.0161 & 1.0010 & 1.0000 & 0.0500 & 0.0032 \\
1.0718 & 0.9614 & 1.0500 & 1.0032 & 1.0000 & 0.2322 & 0.0180 \\
1.1508 & 0.9658 & 1.1180 & 1.0078 & 1.0000 & 0.4001 & 0.0296 \\
1.2840 & 0.9589 & 1.2322 & 1.0161 & 1.0000 & 0.6243 & 0.0500 \\
1.4812 & 0.9338 & 1.4001 & 1.0296 & 1.0000 & 0.9038 & 0.0789 \\
1.7462 & 0.8847 & 1.6243 & 1.0500 & 1.0000 & 1.2361 & 0.1180 \\
2.0795 & 0.8074 & 1.9038 & 1.0789 & 1.0000 & 1.0000 & \\
2.4798 & 0.6986 & 2.2361 & 1.1180 & &
\end{tabular}

$$
\begin{aligned}
& -\left(\frac{i}{2}\right)^{2}(E(x, 2-\beta, i)-E(x, 2-\beta,-i)) \\
& \left.\times \sin ^{2} x+i \sin ^{3} x\right),
\end{aligned}
$$

$$
\begin{aligned}
& H_{1}(U(x, t)) \\
& \begin{aligned}
=2 U_{0} U_{1} \overline{U_{0}} & +U_{0}^{2} \overline{U_{1}} \\
=2\left(\operatorname { s i n } x \left(\frac{t^{\alpha}}{\Gamma(\alpha+1)}\right.\right. & \\
& \times\left(\left(\frac{i}{2}\right)^{2}(E(x, 2-\beta, i)-E(x, 2-\beta,-i))\right. \\
& -i \sin x)) \sin x) \\
-\sin ^{2} x( & \frac{t^{\alpha}}{\Gamma(\alpha+1)} \\
& \times\left(\left(\frac{i}{2}\right)^{2}(E(x, 2-\beta, i)-E(x, 2-\beta,-i))\right. \\
=\frac{t^{\alpha}}{\Gamma(\alpha+1)} & \left(\left(\frac{i}{2}\right)^{2}(E(x, 2-\beta, i)-E(x, 2-\beta,-i))\right. \\
& \left.\times \sin ^{2} x-i \sin ^{3} x\right) .
\end{aligned}
\end{aligned}
$$

Substituting (44) and (45) into (43), we obtain

$$
\begin{aligned}
U_{2}(x, t)= & \frac{t^{2 \alpha}}{\Gamma(2 \alpha+1)} \\
& \times\left(\left(\frac{i}{2}\right)^{3}(E(x, 2-2 \beta, i)-E(x, 2-2 \beta,-i))\right.
\end{aligned}
$$

$$
\begin{aligned}
& +\left(\left(\frac{i}{2}\right)(E(x, 2-\beta, i)-E(x, 2-\beta,-i))\right) \\
& -\sin x) .
\end{aligned}
$$

Substituting $U_{0}(x, t), U_{1}(x, t), U_{2}(x, t), \ldots$ into (24), we obtain the following approximate solution to (41) and (42):

$$
\begin{aligned}
& U_{\text {app }}(x, t) \\
& =\sin x+\frac{t^{\alpha}}{\Gamma(\alpha+1)} \\
& \quad \times\left(\left(\frac{i}{2}\right)^{2}(E(x, 2-\beta, i)-E(x, 2-\beta,-i))-i \sin x\right) \\
& +\frac{t^{2 \alpha}}{\Gamma(2 \alpha+1)} \\
& \times\left(\left(\frac{i}{2}\right)^{3}(E(x, 2-2 \beta, i)-E(x, 2-2 \beta,-i))\right. \\
& \left.\quad+\left(\left(\frac{i}{2}\right)(E(x, 2-\beta, i)-E(x, 2-\beta,-i))\right)-\sin x\right) \\
& +\cdots \\
& =\sin x\left[1+\frac{\left(-i t^{\alpha}\right)}{\Gamma(\alpha+1)}+\frac{\left(-i t^{\alpha}\right)^{2}}{\Gamma(2 \alpha+1)}+\cdots\right] \\
& +\left[\frac{t^{\alpha}}{\Gamma(\alpha+1)}\left(\left(\frac{i}{2}\right)^{2}(E(x, 2-\beta, i)-E(x, 2-\beta,-i))\right)\right. \\
& \quad+\frac{t^{2 \alpha}}{\Gamma(2 \alpha+1)}
\end{aligned}
$$




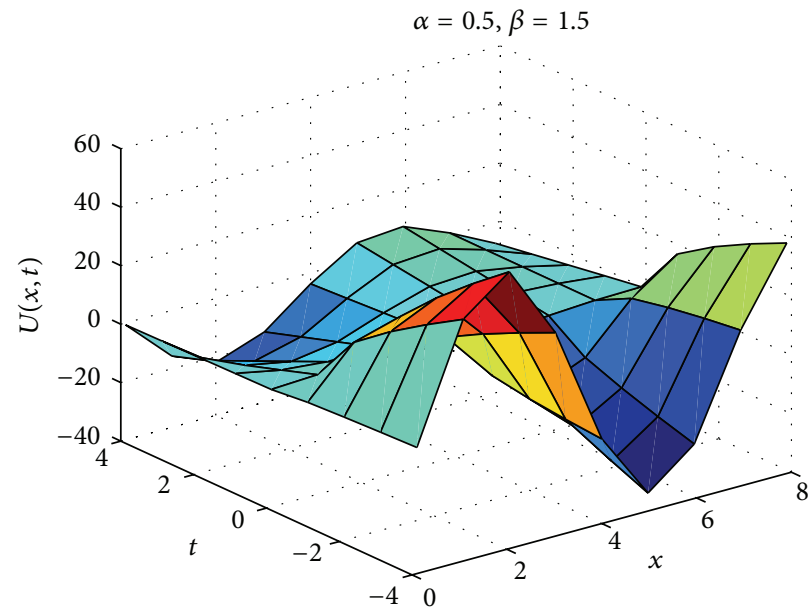

(a)

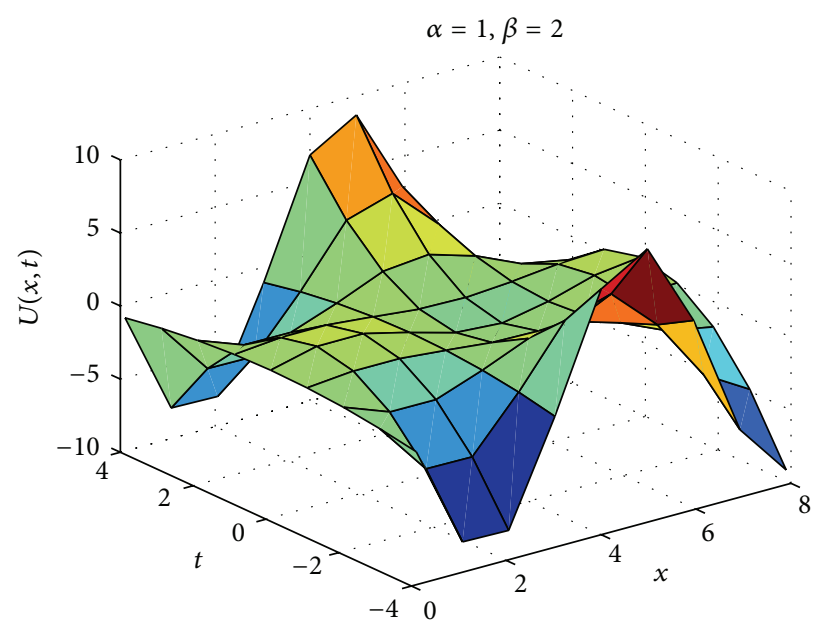

(b)

FIGURE 5: The surface plot of the real part of the approximate solution of Example 11 (a) for $\alpha=0.5, \beta=1.5$ and (b) for the exact solution $\alpha=1, \beta=2$.

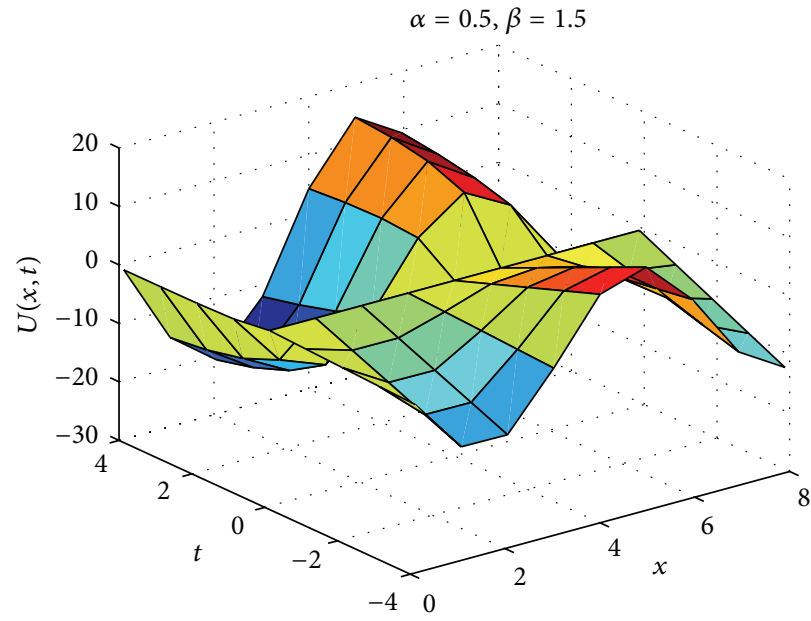

(a)

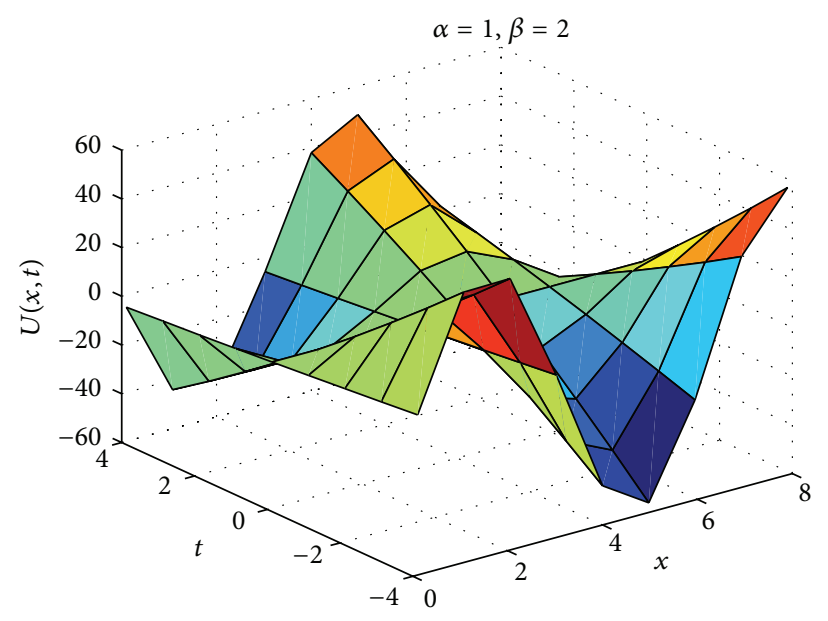

(b)

Figure 6: The surface plot of the imaginary part of the approximate solution of Example 11 (a) for $\alpha=0.5, \beta=1.5$ and (b) for the exact solution $\alpha=1, \beta=2$.

$$
\begin{aligned}
& \times\left(\left(\frac{i}{2}\right)^{3}(E(x, 2-2 \beta, i)-E(x, 2-2 \beta,-i))\right. \\
& \left.\left.\quad+\left(\left(\frac{i}{2}\right)(E(x, 2-\beta, i)-E(x, 2-\beta,-i))\right)\right)+\cdots\right]
\end{aligned}
$$

Figure 5 compares the surface plot of the real part of the approximate solution of Example 11. In Figure 5(a) we have the solution obtained for the values of $\alpha=0.5, \beta=1.5$ and in Figure 5(b) for the exact solution in case $\alpha=1, \beta=2$. Figure 6 compares the surface plot of the imaginary part of the approximate solution of Example 11. In Figure 6(a) we have the solution obtained for the values of $\alpha=0.5, \beta=1.5$ and in Figure 6(b) for the exact solution in case $\alpha=1, \beta=2$.

\section{Conclusion and Discussion}

Homotopy perturbation Sumudu transform method is applied successfully for finding exact solutions for linear space-time fractional Schrödinger equation and approximate solutions for nonlinear fractional Schrödinger equation with space and time fractional derivatives that are considered in the Caputo sense. We have demonstrated the efficiency of this method by four numerical expository examples for a variety of linear and nonlinear space-time fractional Schrödinger equations with zero and nonzero trapping potential. Example 4 is a time fractional nonlinear Schrödinger equation, in which we readily obtained the exact solution in a compact form. Furthermore, the solution is given for the values of $\alpha=$ 0.5 and $\alpha=1$, which are illustrated graphically in Figure 1. Example 6 is a linear space-time fractional Schrödinger 
equation with zero trapping potential, in which we obtained the solution in terms of Mittag-Leffler function; besides, the real and imaginary parts of the solution for the value of $\alpha=$ $0.5, \beta=1.5$ are compared with classical solution graphically in Figures 2 and 3, respectively. In Example 9, the solution of a nonlinear space-time fractional Schrödinger equation with zero trapping potential is approximated and the comparison of the absolute approximate solutions between the homotopy analysis method [29], the homotopy perturbation Sumudu transform method used in this paper, and the exact solution is given in Table 1. It is shown that, for a sufficiently small number of components, the approximate solution given by homotopy perturbation Sumudu transform method becomes nearly more identical to the exact solution than the homotopy analysis method [29]. Example 11 calculated the approximate analytical solution of a nonlinear space-time fractional Schrödinger equation with nonzero trapping potential. To the best of our knowledge, the approximate solution for nonlinear space-time fractional Schrödinger equation with nonzero trapping potential has not been reported in the literature by using the homotopy perturbation method, the Adomian decomposition method, the variational iteration method, and the differential transform method. In conclusion, homotopy perturbation Sumudu transform method is reliable, effective, and easy to implement and produces accurate results. Thus, the method can be applied to solve other nonlinear fractional partial differential equations.

\section{Conflict of Interests}

The authors declare that there is no conflict of interests regarding the publication of this paper.

\section{References}

[1] K. B. Oldham and J. Spanier, The Fractional Calculus, Academic Press, San Diego, Calif, USA, 1974.

[2] K. S. Miller and B. Ross, An Introduction to the Fractional Calculus and Fractional Differential Equations, John Wiley \& Sons, New York, NY, USA, 1993.

[3] S. G. Samko, A. A. Kilbas, and O. I. Marichev, Fractional Integrals and Derivatives: Theory and Applications, Gordon and Breach Science Publishers, Yverdon, Switzerland, 1993.

[4] I. Podlubny, Fractional Differential Equations, Academic Press, San Diego, Calif, USA, 1999.

[5] A. A. Kilbas, H. M. Srivastava, and J. J. Trujillo, Theory and Applications of Fractional Differential Equations, vol. 204, Elsevier, Amsterdam, The Netherlands, 2006.

[6] M. D. Ortigueira, Fractional Calculus for Scientists and Engineers, Springer Science, Berlin, Germany, 2011.

[7] S. Das, Functional Fractional Calculus, Springer, Berlin, Germany, 2nd edition, 2011.

[8] R. Hilfer, Applications of Fractional Calculus in Physics, World Scientific, River Edge, NJ, USA, 2000.

[9] B. J. West, M. Bologna, and P. Grigolini, Physics of Fractal Operators, Springer, New York, NY, USA, 2003.

[10] L. Debnath, "Recent applications of fractional calculus to science and engineering," International Journal of Mathematics and Mathematical Sciences, no. 54, pp. 3413-3442, 2003.
[11] F. Mainardi, Fractional Calculus and Waves in Linear Viscoelasticity: An Introduction to Mathematical Models, Imperial College Press, London, UK, 2010.

[12] D. Baleanu, Z.B. güvenç, and J.A. Tenreiro Machado, New Trends in Nanotechnology and Fractional Calculus Applications, Springer, Berlin, Germany, 2010.

[13] R. Hermann, Fractional Calculus: An Introduction for Physicists, World Scientific, River Edge, NJ, USA, 2011.

[14] S. Momani and Z. Odibat, "Homotopy perturbation method for nonlinear partial differential equations of fractional order," Physics Letters A, vol. 365, no. 5-6, pp. 345-350, 2007.

[15] Q. Wang, "Homotopy perturbation method for fractional KdVBurgers equation," Chaos, Solitons and Fractals, vol. 35, no. 5, pp. 843-850, 2008.

[16] P. K. Gupta and M. Singh, "Homotopy perturbation method for fractional Fornberg-Whitham equation," Computers and Mathematics with Applications, vol. 61, no. 2, pp. 250-254, 2011.

[17] S. S. Ray, "Analytical solution for the space fractional diffusion equation by two-step Adomian decomposition method," Communications in Nonlinear Science and Numerical Simulation, vol. 14, no. 4, pp. 1295-1306, 2009.

[18] S. Z. Rida, H. M. El-Sherbiny, and A. A. M. Arafa, "On the solution of the fractional nonlinear Schrödinger equation," Physics Letters A, vol. 372, no. 5, pp. 553-558, 2008.

[19] M. A. E. Herzallah and K. A. Gepreel, "Approximate solution to the time-space fractional cubic nonlinear Schrodinger equation," Applied Mathematical Modelling. Simulation and Computation for Engineering and Environmental Systems, vol. 36, no. 11, pp. 5678-5685, 2012.

[20] V. S. Ertürk, Z. M. Odibat, and S. Momani, "An approximate solution of a fractional order differential equation model of human T-cell lymphotropic Virus I ( HTLV-I) infection of $\mathrm{CD}^{+}$T-cell," Computers \& Mathematics with Applications, vol. 62, no. 3, pp. 996-1002, 2011.

[21] D. Nazari and S. Shahmorad, "Application of the fractional differential transform method to fractional-order integrodifferential equations with nonlocal boundary conditions," Journal of Computational and Applied Mathematics, vol. 234, no. 3, pp. 883-891, 2010.

[22] F. A. Alawad, E. A. Yousif, and A. I. Arbab, "A new technique of Laplace variational iteration method for solving spacetime fractional telegraph equations," International Journal of Differential Equations, vol. 2013, Article ID 256593, 10 pages, 2013.

[23] J. Lin and G. Hou, "Numerical Coupled Burgers equations by generalized differential transform method," Applied Mathematics and Computation, vol. 217, pp. 7001-7008, 2010.

[24] M. Kurulay and M. Bayram, "Approximate analytical solution for the fractional modified $\mathrm{KdV}$ by differential transform method," Communications in Nonlinear Science and Numerical Simulation, vol. 15, no. 7, pp. 1777-1782, 2010.

[25] P. K. Gupta, "Approximate analytical solutions of fractional Benney-Lin equation by reduced differential transform method and the homotopy perturbation method," Computers and Mathematics with Applications, vol. 61, no. 9, pp. 2829-2842, 2011.

[26] E. A. Abdel-Salam and E. A. Yousif, "Solution of nonlinear space-time fractional differential equations using the fractional Riccati expansion method," Mathematical Problems in Engineering, vol. 2013, Article ID 846283, 6 pages, 2013.

[27] S. Zhang and H. Q. Zhang, "Fractional sub-equation method and its applications to nonlinear fractional PDEs," Physics Letters A, vol. 375, no. 7, pp. 1069-1073, 2011. 
[28] S. Guo, L. Mei, Y. Li, and Y. Sun, "The improved fractional sub-equation method and its applications to the space-time fractional differential equations in fluid mechanics," Physics Letters A, vol. 376, no. 4, pp. 407-411, 2012.

[29] K. M. Hemida, K. A. Gepreel, and M. S. Mohamed, "Analytical approximate solution to the time-space nonlinear partial fractional differential equations," International Journal of Pure and Applied Mathematics, vol. 78, no. 2, pp. 233-243, 2012.

[30] K. A. Gepreel and M. S. Mohamed, "Analytical approximate solution for nonlinear space-time fractional Klein-Gordon equation," Chinese Physics B, vol. 22, no. 1, Article ID 010201, pp. 010201-010211, 2013.

[31] R. Yulita Molliq and B. Batiha, "Approximate analytic solutions of fractional Zakharov-Kuznetsov equation by fractional complex transform," International Journal of Engineering and Technology, vol. 1, no. 1, pp. 1-13, 2012.

[32] G. K. Watugala, "Sumudu transform: a new integral transform to solve differential equations and control engineering problems," International Journal of Mathematical Education in Science and Technology, vol. 24, no. 1, pp. 35-43, 1993.

[33] M. A. Asiru, "Sumudu transform and the solution of integral equations of convolution type," International Journal of Mathematical Education in Science and Technology, vol. 32, no. 6, pp. 906-910, 2001.

[34] A. Kilcman and H. Eltayeb, "A note on integral transforms and partial differential equations," Applied Mathematical Sciences, vol. 4, no. 1-4, pp. 109-118, 2010.

[35] H. Eltayeb and A. K1lıcman, "On some applications of a new integral transform," International Journal of Mathematical Analysis, vol. 4, no. 1-4, pp. 123-132, 2010.

[36] A. Kilcman and H. Eltayeb, "Some remarks on the Sumudu and Laplace transforms and applications to differential equations," ISRN Applied Mathematics, vol. 2012, Article ID 591517, 13 pages, 2012.

[37] A. Kilcman, H. Eltayeb, and K. A. M. Atan, "A note on the comparison between Laplace and Sumudu transforms," Bulletin of the Iranian Mathematical Society, vol. 37, no. 1, pp. 131-141, 2011.

[38] V. G. Gupta and B. Sharma, "Application of Sumudu transform in reaction-diffusion systems and nonlinear waves," Applied Mathematical Sciences, vol. 4, no. 9, pp. 435-446, 2010.

[39] M. A. Rana, A. M. Siddiqui, Q. K. Ghori, and R. Qamar, "Application of He's homotopy perturbation method to sumudu transform," International Journal of Nonlinear Sciences and Numerical Simulation, vol. 8, no. 2, pp. 185-190, 2007.

[40] V. Chaurasia and J. Singh, "Application of Sumudu transform in fractional kinetic equations," General Mathematics Notes, vol. 2, no. 1, pp. 86-95, 2011.

[41] D. Kumar and J. Singh, "Sumudu homotopy perturbation technique," Global Journal of Science Frontier Research, vol. 11, no. 6, 2011.

[42] H. Bulut, H. Baskonus, and S. Tulue, "Homotopy perturbation Sumudu transform method for heat equations," Mathematics in Engineering, Science \& Aerospace (MESA), vol. 4, no. 1, p. 49, 2013.

[43] J. Singh, D. Kumar, and A. Kilicman, "Application of homotopy perturbation Sumudu transform method for solving heat and wave-like equations," Malaysian Journal of Mathematical Sciences, vol. 7, no. 1, pp. 79-95, 2013.

[44] S. Rathore, D. Kumer, J. Singh, and S. Gupta, "Homotopy analysis Sumudu transform method for nonlinear equations,"
International Journal of Industrial Mathematics, vol. 4, no. 4, 13 pages, 2012.

[45] A. Atangana and A. Kılıçman, "The use of Sumudu transform for solving certain nonlinear fractional heat-like equations," Abstract and Applied Analysis, vol. 2013, Article ID 737481, 12 pages, 2013.

[46] J. Singh, D. Kumar, and A. Kılıçman, "Homotopy perturbation method for fractional gas dynamics equation using Sumudu transform," Abstract and Applied Analysis, vol. 2013, Article ID 934060, 8 pages, 2013.

[47] A. A. Elbeleze, A. Kılıçman, and B. M. Taib, "Homotopy perturbation method for fractional Black-Scholes European option pricing equations using SUMudu transform," Mathematical Problems in Engineering, vol. 2013, Article ID 524852, 7 pages, 2013.

[48] L. Vázquez, L. Streit, and V. M. Pérez-García, Eds., Proceedings of the Euroconference on Nonlinear Klein-Gordon and Schrödinger Systems: Theory and Applications, World Scientific, Singapore, 1997.

[49] Y. Kivshar and G. P. Agrawal, Optical Solitons: From Fibers to Photonic Crystals, Academic Press, 2003.

[50] R. Fedele, G. Miele, L. Palumbo, and V. G. Vaccaro, "Thermal wave model for nonlinear longitudinal dynamics in particle accelerators," Physics Letters A, vol. 179, no. 6, pp. 407-413, 1993.

[51] F. Dalfovo, S. Giorgini, L. P. Pitaevskii, and S. Stringari, “Theory of Bose-Einstein condensation in trapped gases," Reviews of Modern Physics, vol. 71, no. 3, pp. 463-512, 1999.

[52] J. Belmonte-Beitia and G. F. Calvo, "Exact solutions for the quintic nonlinear Schrödinger equation with time and space modulated nonlinearities and potentials," Physics Letters A, vol. 373, no. 4, pp. 448-453, 2009.

[53] T. Xu, B. Tian, L. L. Li, X. Lu, and C. Zhang, "Dynamics of Alfvén solitons in inhomogeneous plasmas," Physics Plasmas, vol. 15, Article ID 102307, 2008.

[54] M. Naber, "Time fractional Schrödinger equation," Journal of Mathematical Physics, vol. 45, no. 8, pp. 3339-3352, 2004.

[55] S. Wang and M. Xu, "Generalized fractional Schrödinger equation with space-time fractional derivatives," Journal of Mathematical Physics, vol. 48, no. 4, Article ID 043502, 10 pages, 2007.

[56] X. Y. Jiang, “Time-space fractional Schrödinger like equation with a nonlocal term," European Physical Journal: Special Topics, vol. 193, no. 1, pp. 61-70, 2011.

[57] N. J. Ford, M. M. Rodrigues, and N. Vieira, "A numerical method for the fractional Schrödinger type equation of spatial dimension two," Fractional Calculus and Applied Analysis, vol. 16, no. 2, pp. 454-468, 2013.

[58] B. Guo, Y. Han, and J. Xin, "Existence of the global smooth solution to the period boundary value problem of fractional nonlinear Schrödinger equation," Applied Mathematics and Computation, vol. 204, no. 1, pp. 468-477, 2008.

[59] J. Hu, J. Xin, and H. Lu, "The global solution for a class of systems of fractional nonlinear Schrödinger equations with periodic boundary condition," Computers \& Mathematics with Applications, vol. 62, no. 3, pp. 1510-1521, 2011.

[60] R. K. Saxena and S. L. Kalla, "Computational solution of a fractional generalization of the Schrodinger equation occurring in quantum mechanics," Applied Mathematics and Computation, vol. 216, no. 5, pp. 1412-1417, 2010.

[61] J. Wang, Y. Zhou, and W. Wei, "Fractional Schrödinger equations with potential and optimal controls," Nonlinear Analysis: Real World Applications, vol. 13, no. 6, pp. 2755-2766, 2012. 
[62] N. A. Khan, M. Jamil, and A. Ara, "Approximate solutions to time-fractional Schrödinger equation via homotopy analysis method," ISRN Mathematical Physics, vol. 2012, Article ID 197068, 11 pages, 2012.

[63] H. J. Haubold, A. M. Mathai, and R. K. Saxena, "MittagLeffler functions and their applications," Journal of Applied Mathematics, vol. 2011, Article ID 298628, 51 pages, 2011.

[64] A. A. Kilbas, M. Saigo, and R. K. Saxena, "Generalized mittagleffler function and generalized fractional calculus operators," Integral Transforms and Special Functions, vol. 15, no. 1, pp. 3149, 2004.

[65] J. He, "Homotopy perturbation technique," Computer Methods in Applied Mechanics and Engineering, vol. 178, no. 3-4, pp. 257$262,1999$.

[66] J. H. He, "Recent development of the homotopy perturbation method," Topological Methods in Nonlinear Analysis, vol. 31, no. 2, pp. 205-209, 2008.

[67] J. He, "A modified perturbation technique depending upon an artificial parameter," Meccanica, vol. 35, no. 4, pp. 299-311, 2000. 


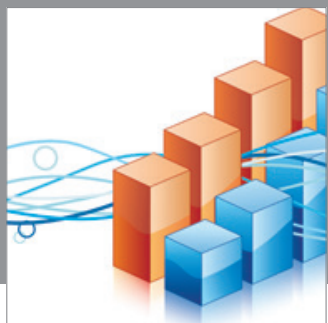

Advances in

Operations Research

mansans

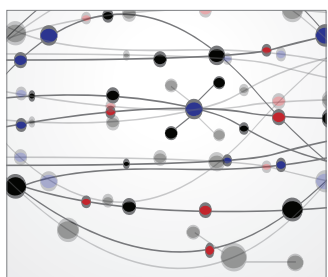

The Scientific World Journal
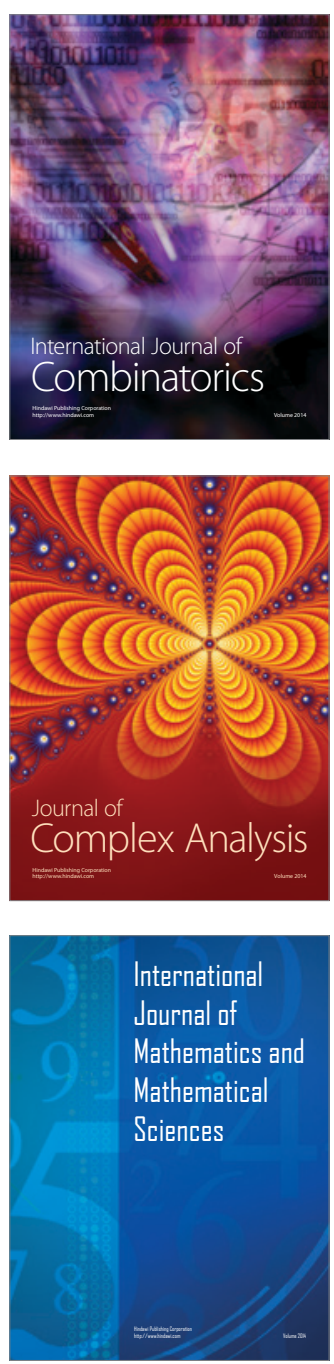
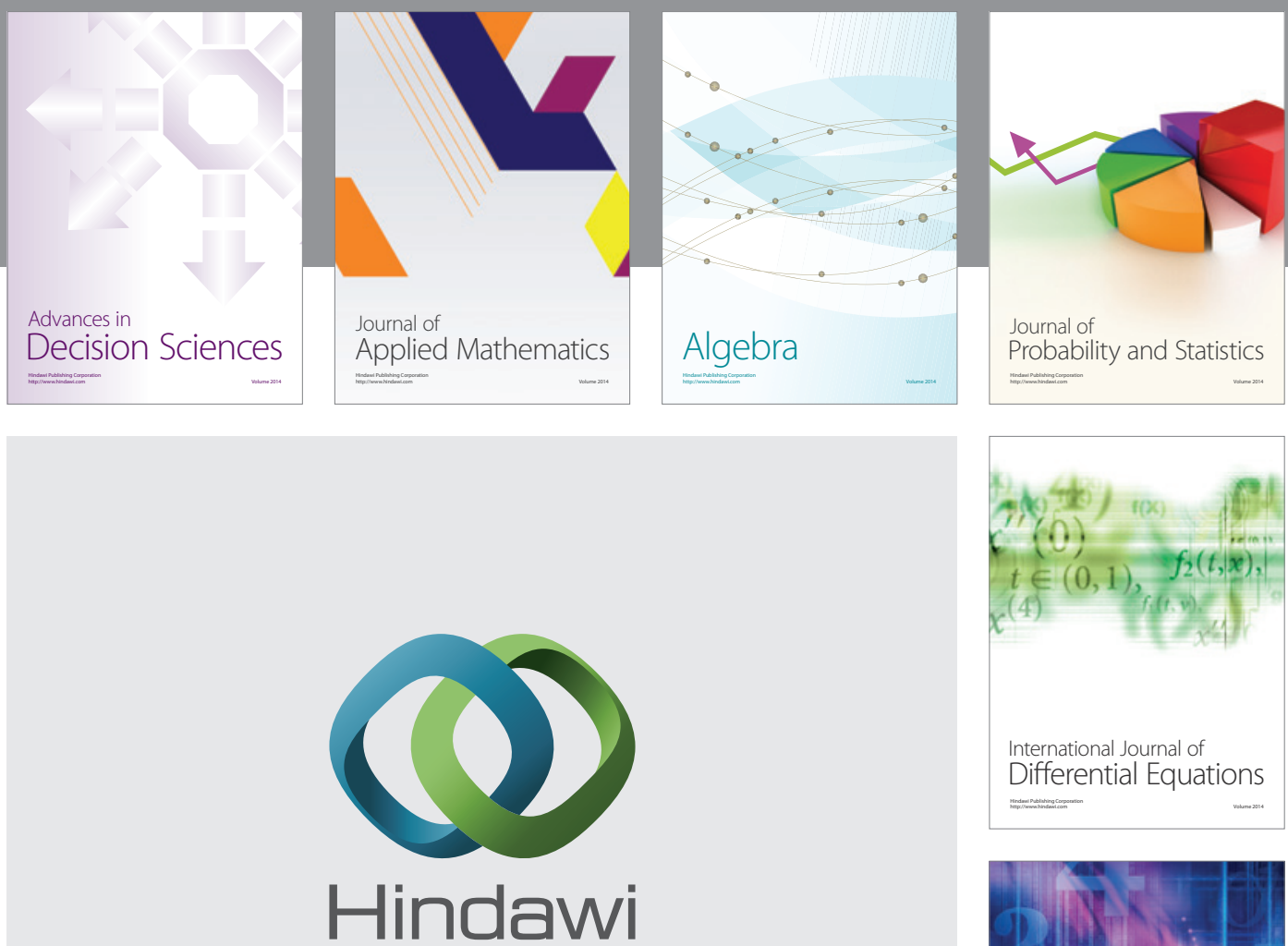

Submit your manuscripts at http://www.hindawi.com
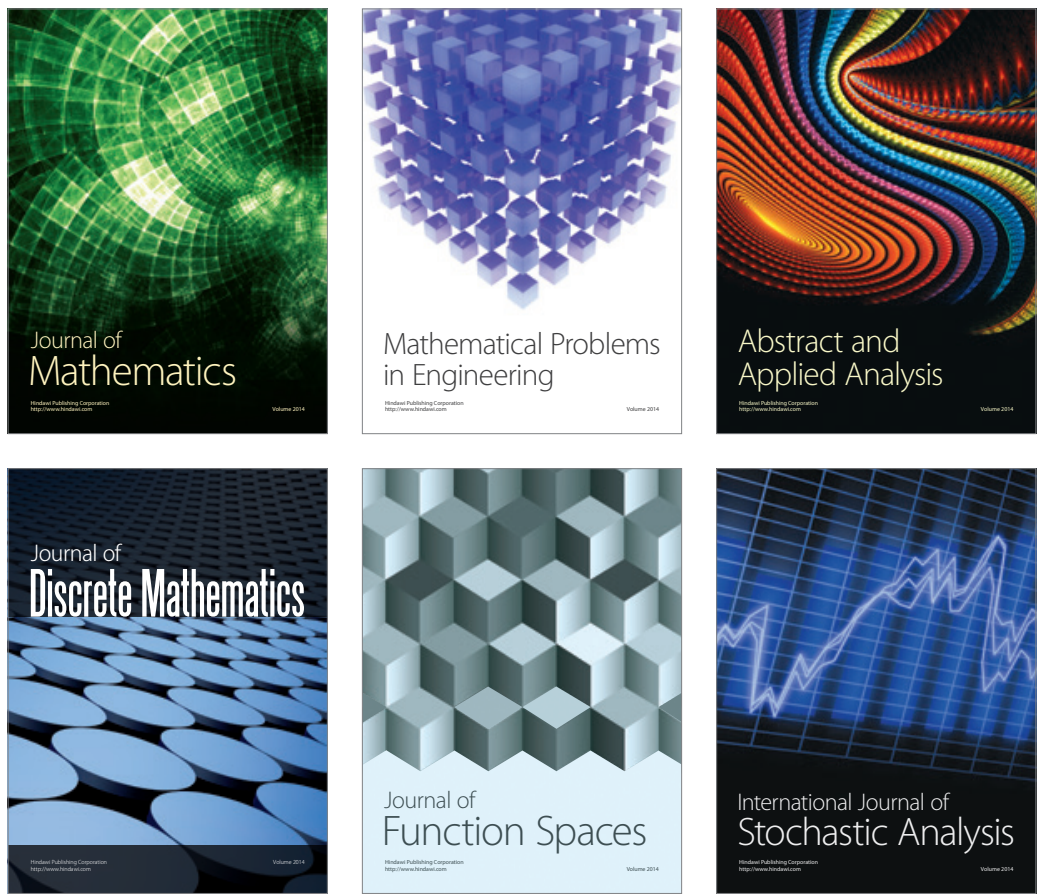

Journal of

Function Spaces

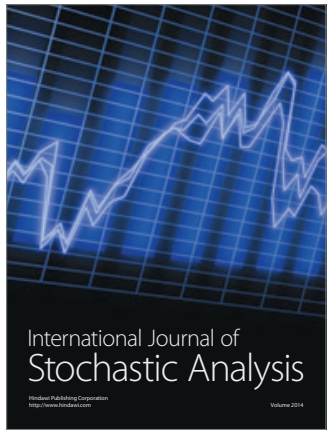

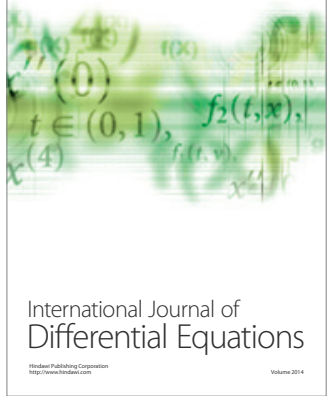
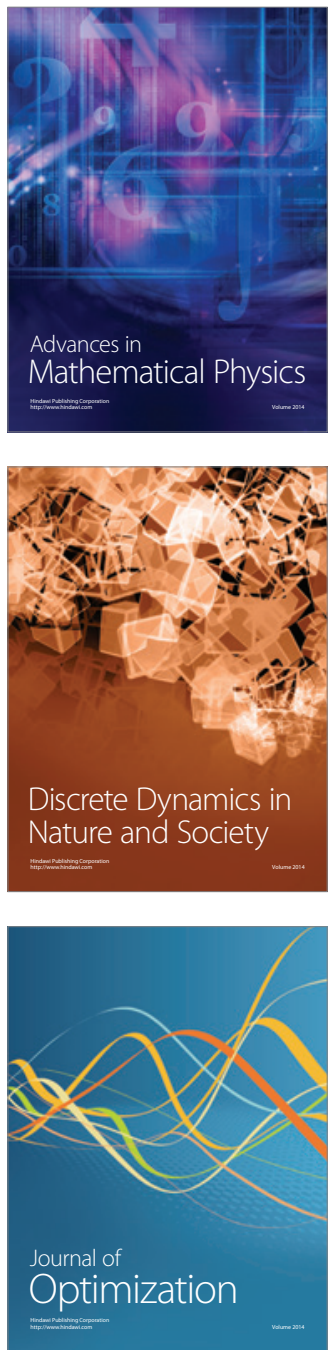